\title{
sciendo
}

\section{How do Amateur Endurance Runners Alter Spatiotemporal Parameters and Step Variability as Running Velocity Increases? A Sex Comparison}

\author{
by \\ Felipe García-Pinillos ${ }^{1}$, Daniel Jerez-Mayorga², Pedro Á. Latorre-Román ${ }^{3}$, \\ Rodrigo Ramirez-Campillo ${ }^{4}$ Fernando Sanz-López ${ }^{5}$, Luis E. Roche-Seruendo ${ }^{6}$
}

This study aimed to analyse the effects of running velocity on spatiotemporal parameters and step variability in amateur endurance runners, according to sex. A group of 51 males and 46 females performed an incremental running test on a treadmill $(10-16 \mathrm{~km} / \mathrm{h})$. Spatiotemporal parameters (contact and flight time, step length, step frequency and step angle [CT, FT, SL, SF, SA]) and step variability, in terms of within-participant standard deviation $(S D)$, were recorded through the OptoGait System. The ANOVA showed significant differences in the magnitude of the spatiotemporal parameters as running velocity increased $(p<0.001)$. It also revealed significant differences in step variability $(p<0.005)$ over the entire running protocol. Between-sex differences in CT, SL, SL-normalized and SF ( $p<$ $0.05, E S=0.4-0.8)$ were found. Differences were also found in step variability at high velocities $(15-16 \mathrm{~km} / \mathrm{h})$, with males showing a greater SD than females. In conclusion, increasing running velocity makes CT shorter, FT and SL longer, and SF and SA greater in amateur endurance runners, changing step variability, regardless of sex. Additionally, some between-sex differences were found in spatiotemporal parameters and step variability.

Key words: gender, biomechanics, kinematics, running.

\section{Introduction}

The influence of running velocity on spatiotemporal gait characteristics has been previously examined in the literature (Brughelli et al., 2010; Ogueta-Alday et al., 2014; Padulo et al., 2012; Roche-Seruendo et al., 2018). The overall decrease in the contact time (CT) and the increase in flight time (FT), step length (SL), and step frequency (SF) with increasing running speed have been previously shown (Brughelli et al., 2010; Ogueta-Alday et al., 2014; Padulo et al., 2012; Roche-Seruendo et al., 2018). However, new

devices for examining running biomechanics have arisen (e.g., OptoGait ${ }^{\mathrm{TM}}$ system) facilitating the assessment of new parameters, e.g., step angle [SA] (Santos-Concejero et al., 2014) and step variability (García-Pinillos et al., 2018a). Moreover, there is limited evidence about the dynamics of these parameters in amateur endurance runners as running velocity increases, especially regarding the interpretation of these parameters.

According to a previous study (Santos-

\footnotetext{
1 - Department of Physical Education, Sports and Recreation. Universidad de La Frontera (Temuco, Chile).

2 - Faculty of Rehabilitation Sciences. Universidad Andres Bello. (Santiago. Chile).

3 - Department of Corporal Expression. University of Jaen (Jaen, Spain).

4 - Laboratory of Human Performance. Quality of Life and Wellness Research Group. Department of Physical Activity Sciences. Universidad de Los Lagos (Osorno, Chile).

5 - Aspetar Orthopaedic and Sports Medicine Hospital. Physical Therapy Department (Doha, Qatar).

6 - Department of Physiotherapy. Universidad San Jorge (Zaragoza, Spain).
} 
Concejero et al., 2014), the SA is an easily obtainable measure during running, allowing an indirect biomechanical assessment of the flight phase and vertical ground contact forces. In order to support that statement, it has been shown that less economical runners exhibit greater total and net vertical impulses, indicating wasteful vertical motion (Heise and Martin, 2001), which has been strongly associated with a greater SA (SantosConcejero et al., 2014). Some previous studies have investigated the SA during running and its adaptations to changes in slope gradients (GarcíaPinillos et al., 2018b) or different percentages of body weight support (Roche-Seruendo et al., 2017), but no studies have considered the effect of running velocity on the SA.

Not only may the magnitude of the aforementioned spatiotemporal parameters be important, but also its variability. Step variability provides a description of the amount of variability in the time series, and alterations are generally considered evidence for changes in the stability of the coordination pattern (Jordan et al., 2009). Some studies have suggested a relationship between step variability and both injuries (Hamill et al., 2012; Meardon et al., 2011) and endurance performance (Nakayama et al., 2010). Despite the importance of step variability during running, the evidence about potential influencing factors such as running velocity is quite limited (GarcíaPinillos et al., 2018a). A previous study (GarcíaPinillos et al., 2018a) described the step variability during running at different submaximal velocities (i.e., 10, 12, 14 and $16 \mathrm{~km} / \mathrm{h}$ ), although did not assess the impact of running velocity on the magnitude of step variability.

Additionally, since some between-sex differences are generally observed in body size and proportions, and body structure has been associated to spatiotemporal characteristics during running (Kong and de Heer, 2008; RocheSeruendo et al., 2019), it seems that sex might be an influencing factor for the spatiotemporal adaptations during running with increased velocity. Nevertheless, no previous studies have described spatiotemporal differences between sexes in endurance runners.

Considering the lack of information about the dynamics of the SA and step variability with increasing running velocity according to sex, the aim of this research was twofold: (i) to determine the effects of running velocity on spatiotemporal parameters and step variability in amateur endurance runners; and (ii) to determine the sex influence on those spatiotemporal adaptations experienced during an incremental running test.

\section{Methods}

Endurance runners performed an incremental running test. An analysis of spatiotemporal gait characteristics and step variability (in terms of within-participant standard deviation [SD]) over the entire protocol was conducted, as also with the aim to determine the influence of sex on those adaptations. A unilateral crossover design was used, with all athletes performing the same protocol under the same conditions.

\section{Participants}

Amateur endurance runners (51 males, 46 females; age: $27 \pm 8$ years; age range: 18 - 40 years; body height: $172 \pm 9 \mathrm{~cm}$; body mass: $66 \pm 10 \mathrm{~kg}$ ), able to run $10-\mathrm{km}$ in less than 45 minutes (42.1 \pm $1.8 \mathrm{~min})$, participated in this study. Participants met the inclusion criteria: (i) $\geq 18$ years old; (ii) recreationally active (3-4 running sessions per week, at least once on a treadmill); (iii) have not suffered from any injury within the last 6 months before data collection. After receiving detailed information on the objectives and procedures of the study, each participant signed an informed consent form, which complied with the ethical standards of the World Medical Association's Declaration of Helsinki (2013); it was made clear that participants were free to withdraw from the study at any moment. The University of San Jorge ethics committee approved the study.

\section{Procedures}

Before the running protocol, participants performed a warm-up, with $5 \mathrm{~min}$ of continuous running and 5 min of active joint mobilization and dynamic stretching. Then, participants performed an incremental running test protocol on a treadmill (Salter M-835, Salter Int., Barcelona, Spain). The running protocol was preceded by an adaptation period of $10 \mathrm{~min}$ at a steady pace of 10 $\mathrm{km} / \mathrm{h}$. Participants were familiar with running on a treadmill, although previous studies (Lavcanska et al., 2005) on human locomotion have shown that accommodation to running on a treadmill occurs within 6-8 min. The running test started at $10 \mathrm{~km} / \mathrm{h}$ and running speed was increased $1 \mathrm{~km} / \mathrm{h}$ 
every $20 \mathrm{~s}(10 \mathrm{~s}$ acclimatization period: $10 \mathrm{~s}$ recording period), finishing at $16 \mathrm{~km} / \mathrm{h}$. Participants, therefore, completed an incremental test with 7 different running velocities (10-16 $\mathrm{km} / \mathrm{h}$ ). The short duration of each running speed aimed to minimize the effect of fatigue on running kinematics and allowed participants to complete the test at running speeds at which amateur runners usually trained. This protocol was previously used by Roche-Seruendo et al. (2018). Participants verbally reported feeling comfortable while running on the treadmill at the set speeds.

\section{Materials and testing}

i) Anthropometry. For descriptive purposes, body height $(\mathrm{cm})$ and body mass $(\mathrm{kg})$ were determined using a precision stadiometer and mechanical scale (SECA 222 and 634, respectively, SECA Corp., Hamburg, Germany). All measurements were taken with participants wearing underwear.

ii) Spatiotemporal parameters. The measurements were performed with the OptoGait system (Optogait; Microgate, Bolzano, Italy), which was previously validated for the assessment of gait spatiotemporal parameters of young adults, reporting a high correlation with all spatiotemporal parameters via intra-class correlation coefficients (0.8-1.0), coefficients of variation (1.7-4.1\%), standard error of measurement $(2.2-6.0 \%)$, and minimum detectable change (6.0-16.5\%) (Lee et al., 2014). The two parallel bars of the device system were placed on the side edges of the treadmill at the same level as the contact surface. This device was connected to a computer controlled by the responsible researcher. Data were recorded and averaged for the subsequent analyses. In accordance with the findings from a previous study (Brown et al., 2014), limb dominance was not taken into account. Spatiotemporal parameters were measured for every step during the treadmill protocol as follows:

- Contact time (CT, in seconds [s]): time from when the foot contacted the ground to when the toes lifted off the ground.

- Flight time (FT, in seconds [s]): time from toe-off to initial ground contact of consecutive footfalls (i.e., right-left).

- Step length (SL, in meters [m]): length the treadmill belt moved from toe-off to initial ground contact in successive steps. Based on the anthropometric differences between sexes and similar to previous studies (Alexander, 1984), the SL was normalized according to leg length (SLnormalized).

- Step frequency (SF, in steps per minute [spm]): the number of ground contact events per minute.

- Step angle (SA, in degrees [ $\left.\left.{ }^{\circ}\right]\right)$ : the angle of the parabolic tangent deriving from the SL and the height during FT. The determination of SL is described above, and the maximal height of the foot during a stride was calculated by the Optogait system (SantosConcejero et al., 2014).

iii) Step variability. Step variability was assessed for each spatiotemporal parameter as previously suggested (Nakayama et al., 2010), through the within-participant standard deviation (SD). Step variability was examined over the entire recording period (10 s) at every velocity tested $(10-16 \mathrm{~km} / \mathrm{h})$. As indicated by previous studies (Belli et al., 1995; García-Pinillos et al., 2018a), the number of steps analysed over the recording period (10 s) was $\sim 30 \mathrm{spm}$, which seems to be the minimum number of steps required to accurately estimate spatial and temporal step variability of subjects running on a treadmill at submaximal intensities.

\section{Statistical Analysis}

Descriptive statistics are presented as mean and SD. The normal distribution of data and homogeneity of variances were confirmed through the Kolmogorov-Smirnov and Levene's tests, respectively $(p>0.05)$. A one-way analysis of variance (ANOVA) was conducted to compare the group's characteristics according to sex. A repeated measures ANOVA (sex*measurements), with the Bonferroni post-hoc test, was used to determine the effects of running velocity on spatiotemporal parameters in both sex groups. Repeated measures ANOVA was also conducted to determine whether changes in running velocity caused alterations in step variability (i.e., SD) for each spatiotemporal parameter in both sex groups. Delta $(\Delta)$ was calculated in terms of spatiotemporal changes (in \%) associated to running velocity increases. The magnitude of the differences between values was interpreted using the Cohen's d effect size (ES) (between-group differences) (Cohen, 1988). Effect sizes are reported as: trivial $(<0.2)$, small $(0.2-0.49)$, medium 
(0.5-0.79), and large $(\geq 0.8)$ (Cohen, 1988). Data analysis was performed using SPSS (version 21, SPSS Inc., Chicago, IL, USA), and the level of significance was set at $p<0.05$.

\section{Results}

Table 1 shows the characteristics of participants according to sex. No between-sex differences were found in age or leg length $(p \geq$ $0.05)$, but significant differences were found in body height, body mass, and the BMI $(p<0.001)$.

The ANOVAs showed significant differences in the spatiotemporal parameters as running velocity increased $(10-16 \mathrm{~km} / \mathrm{h})$ (Table 2$)$. The CT decreased $(p<0.001)$, whereas FT, SL, SLnormalized, SF and SA increased $(p<0.001)$ with increasing running velocity. Bonferroni pairwise comparison reported significant differences in each parameter with each increase in velocity, regardless of sex. Regarding the sex comparison, females showed shorter CT than males at every velocity $(10-16 \mathrm{~km} / \mathrm{h}, p<0.05$, ES $=0.46-0.67)$. Additionally, at velocities $\geq 14 \mathrm{~km} / \mathrm{h}$, females showed shorter SL $(p<0.05$, ES $=0.48-0.79)$, SLnormalized $(p<0.05$, ES $=0.40-0.62)$, and higher SF $(p<0.05$, ES $=0.47-0.79)$. No between-sex differences were found in the SA $(p \geq .05$, ES $<$ $0.314)$ nor in FT $(p \geq 0.05$, ES $<0.354)$. Figure 1 illustrates the relative change (\%) in spatiotemporal parameters at each running velocity.

The ANOVAs conducted on the withinparticipants SD (step variability, Table 3 ) revealed significant differences for CT $(p<0.001)$, FT $(p<$ $0.001)$, SL $(p=0.026)$, SLnormalized $(p=0.011)$ and SA $(p<0.001)$ over the incremental running protocol; whereas no significant differences were found in SF $(p=0.38)$. Regardless of sex, the Bonferroni pairwise comparison reported significant differences in each parameter. Males showed greater step variability than females at 15-16 $\mathrm{km} / \mathrm{h}$, with no sex differences at $10-14 \mathrm{~km} / \mathrm{h}(p \geq$ 0.5 , ES <0.5).

\section{Discussion}

This study aimed to investigate the effects of running velocity on spatiotemporal parameters and step variability in amateur endurance runners, as well as to determine the sex influence on those spatiotemporal adaptations experienced during an incremental running test $(10-16 \mathrm{~km} / \mathrm{h})$. The current work provides a detailed description of spatiotemporal parameters dynamics over a range of common training velocities in a meaningful sample $(n=97)$ of amateur male and female endurance runners. As running velocity increased, CT decreased (1-6\%), FT and SL increased $(10-34 \%$ and $6-8 \%$ [ $8-51 \%$ if SLnormalized is considered], respectively), and SF and SA were greater $\quad(1.5-2 \%$ and $10-60 \%$, respectively). Moreover, step variability was dependent on running velocity, with five out of six parameters (CT, FT, SL, SLnormalized and SA) altered with increased velocity. Regarding the sex comparison, females showed shorter CT than males at every velocity tested, shorter SL and SLnormalized at velocities $\geq 14 \mathrm{~km} / \mathrm{h}$, and higher $\mathrm{SF}$, with no differences in the SA. Likewise, between-sex differences were found in step variability at 15-16 $\mathrm{km} / \mathrm{h}$, with males showing greater step variability than females in each parameter.

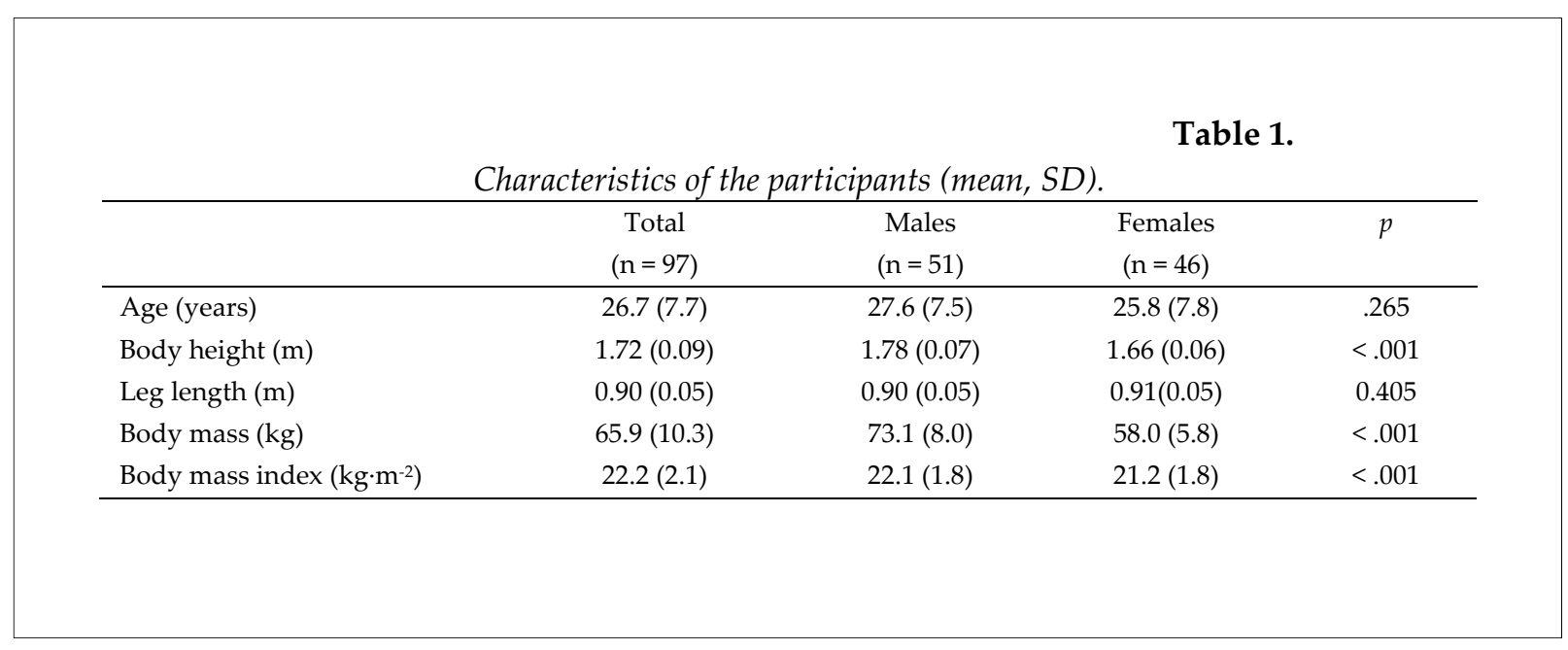




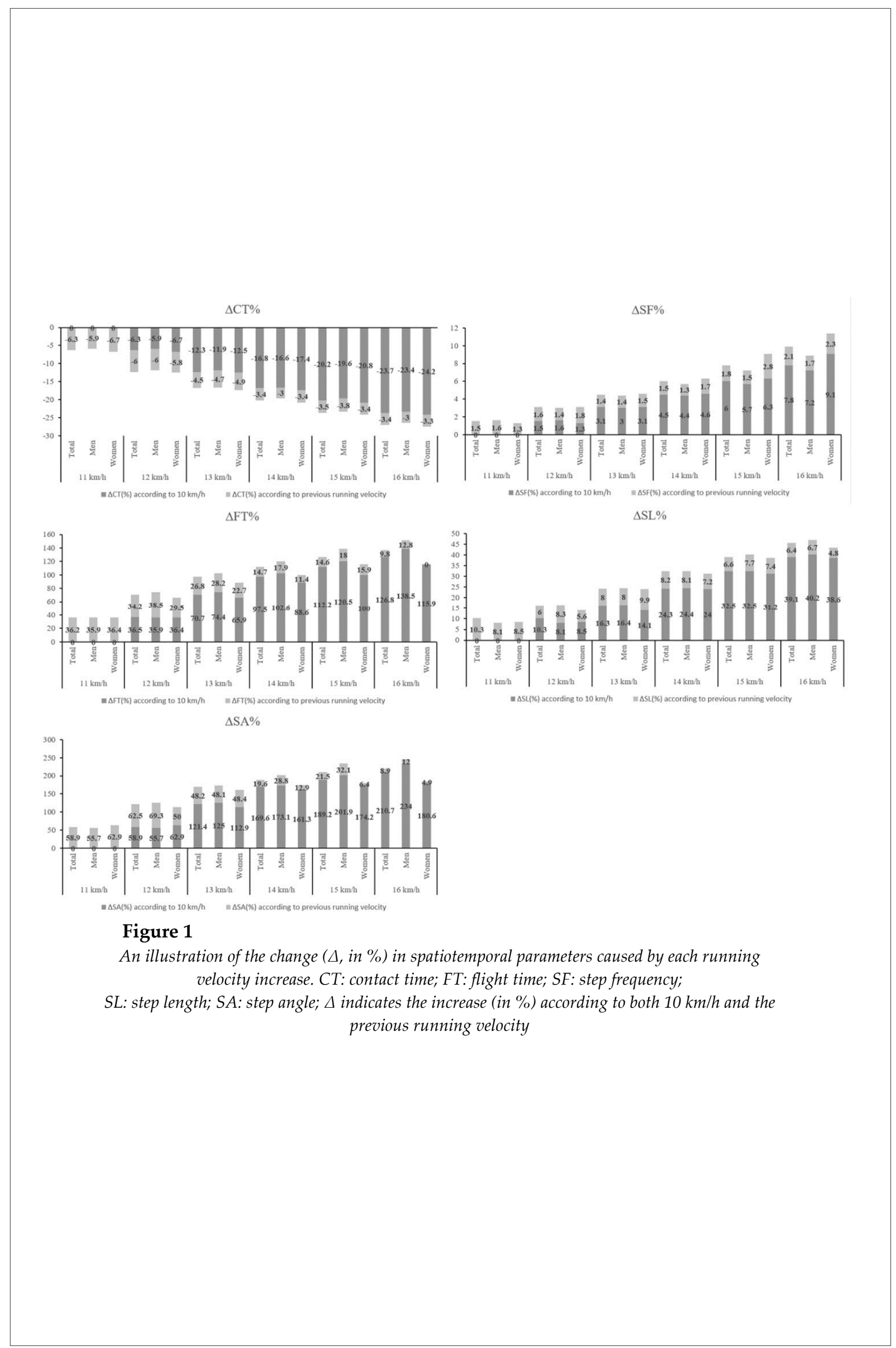




\begin{tabular}{|c|c|c|c|c|c|c|}
\hline \multicolumn{4}{|c|}{$\begin{array}{l}\text { Table } 2 \\
\text { Descriptive values and association observed for the spatiotemporal parameters } \\
(C T, F T, S L, S F \text { and } S A) \text { at every running velocity tested, according to sex. }\end{array}$} & \multicolumn{2}{|c|}{$\begin{array}{l}\text { Table } 2 \\
\text { tiotemporal parameters } \\
\text { sted, according to sex. }\end{array}$} & \multirow[b]{2}{*}{ ES } \\
\hline & & Total $(\mathrm{n}=97)$ & Male $(\mathrm{n}=51)$ & Female $(n=46)$ & $p$ & \\
\hline \multirow{6}{*}{$\begin{array}{c}10 \\
\mathrm{~km} / \mathrm{h}\end{array}$} & $\mathrm{CT}(\mathrm{s})$ & $0.333(0.024)$ & $0.337(0.026)$ & $0.327(0.017)$ & 0.039 & 0.455 \\
\hline & FT (s) & $0.041(0.018)$ & $0.039(0.019)$ & $0.044(0.018)$ & 0.238 & 0.272 \\
\hline & $\mathrm{SL}(\mathrm{cm})$ & $103.84(5.06)$ & $104.54(4.93)$ & $102.88(5.14)$ & 0.141 & 0.333 \\
\hline & SLnormalized & $1.16(0.08)$ & $1.17(0.08)$ & $1.14(0.08)$ & 0.119 & 0.378 \\
\hline & $\mathrm{SF}$ (step/min) & $161.07(7.82)$ & $159.99(7.66)$ & $162.56(7.82)$ & 0.141 & 0.337 \\
\hline & $\mathrm{SA}\left({ }^{\circ}\right)$ & $0.56(0.51)$ & $0.52(0.51)$ & $0.62(0.50)$ & 0.418 & 0.200 \\
\hline \multirow{6}{*}{$\begin{array}{c}11 \\
\mathrm{~km} / \mathrm{h}\end{array}$} & $\mathrm{CT}(\mathrm{s})$ & $0.312(0.022)^{\mathrm{a}}$ & $0.317(0.023)^{\mathrm{a}}$ & $0.305(0.018)^{a}$ & 0.015 & 0.583 \\
\hline & $\mathrm{FT}(\mathrm{s})$ & $0.056(0.020)^{\mathrm{a}}$ & $0.053(0.020)^{\mathrm{a}}$ & $0.060(0.020)^{\mathrm{a}}$ & 0.111 & 0.354 \\
\hline & $\mathrm{SL}(\mathrm{cm})$ & $112.39(5.71)^{\mathrm{a}}$ & $112.96(5.63)^{\mathrm{a}}$ & $111.60(5.81)^{\mathrm{a}}$ & 0.286 & 0.240 \\
\hline & SLnormalized & $1.25(0.09)^{\mathrm{a}}$ & $1.26(0.09)^{\mathrm{a}}$ & $1.24(0.10)^{\mathrm{a}}$ & 0.240 & 0.213 \\
\hline & $\mathrm{SF}$ (step/min) & $163.49(8.00)^{\mathrm{a}}$ & $162.60(7.85)^{\mathrm{a}}$ & $164.72(8.15)^{\mathrm{a}}$ & 0.234 & 0.266 \\
\hline & $\mathrm{SA}(\stackrel{\circ}{)})$ & $0.89(0.63)^{\mathrm{a}}$ & $0.81(0.59)^{\mathrm{a}}$ & $1.01(0.66)^{\mathrm{a}}$ & 0.161 & 0.314 \\
\hline \multirow{6}{*}{$\begin{array}{c}12 \\
\mathrm{~km} / \mathrm{h}\end{array}$} & $\mathrm{CT}(\mathrm{s})$ & $0.292(0.020)^{a, b}$ & $0.297(0.020)^{a, b}$ & $0.286(0.016)^{a, b}$ & 0.010 & 0.610 \\
\hline & $\mathrm{FT}(\mathrm{s})$ & $0.070(0.018)^{a, b}$ & $0.068(0.018)^{a, b}$ & $0.073(0.017)^{a, b}$ & 0.232 & 0.288 \\
\hline & $\mathrm{SL}(\mathrm{cm})$ & $120.88(6.00)^{\mathrm{a}, \mathrm{b}}$ & $121.72(5.92)^{a, b}$ & $119.72(5.99)^{a, b}$ & 0.135 & 0.340 \\
\hline & SLnormalized & $1.34(0.10)^{a, b}$ & $1.36(0.10)^{a, b}$ & $1.33(0.11)^{\mathrm{a}, \mathrm{b}}$ & 0.173 & 0.289 \\
\hline & $\mathrm{SF}$ (step/min) & $166.02(8.22)^{a, b}$ & $164.80(8.13)^{a, b}$ & $167.70(8.15)^{a, b}$ & 0.114 & 0.360 \\
\hline & $\mathrm{SA}\left({ }^{\circ}\right)$ & $1.24(0.60)^{\mathrm{a}, \mathrm{b}}$ & $1.17(0.58)^{\mathrm{a,b}}$ & $1.33(0.62)^{\mathrm{a}, \mathrm{b}}$ & 0.234 & 0.272 \\
\hline \multirow{6}{*}{$\begin{array}{c}13 \\
\mathrm{~km} / \mathrm{h}\end{array}$} & $\mathrm{CT}(\mathrm{s})$ & $0.277(0.019)^{\mathrm{a}, \mathrm{b}, \mathrm{c}}$ & $0.281(0.019)^{\mathrm{a}, \mathrm{b}, \mathrm{c}}$ & $0.270(0.017)^{\mathrm{a}, \mathrm{b}, \mathrm{c}}$ & 0.009 & 0.615 \\
\hline & $\mathrm{FT}(\mathrm{s})$ & $0.081(0.018)^{\mathrm{a}, \mathrm{b}, \mathrm{c}}$ & $0.079(0.018)^{\mathrm{a}, \mathrm{b}, \mathrm{c}}$ & $0.083(0.017)^{\mathrm{a}, \mathrm{b}, \mathrm{c}}$ & 0.263 & 0.230 \\
\hline & $\mathrm{SL}(\mathrm{cm})$ & $129.02(6.69)^{\mathrm{a}, \mathrm{b}, \mathrm{c}}$ & $130.03(6.88)^{\mathrm{a}, \mathrm{b}, \mathrm{c}}$ & $127.63(6.25)^{a, b, c}$ & 0.106 & 0.367 \\
\hline & SLnormalized & $1.43(0.11)^{a, b, c}$ & $1.45(0.11)^{a, b, c}$ & $1.42(0.12)^{a, b, c}$ & 0.186 & 0.263 \\
\hline & $\mathrm{SF}$ (step/min) & $168.25(8.53)^{a, b, c}$ & $166.97(8.71)^{\mathrm{a}, \mathrm{b}, \mathrm{c}}$ & $170.00(8.05)^{\mathrm{a}, \mathrm{b}, \mathrm{c}}$ & 0.111 & 0.362 \\
\hline & SA $\left({ }^{\circ}\right)$ & $1.51(0.62)^{\mathrm{a}, \mathrm{b}, \mathrm{c}}$ & $1.43(0.60)^{a, b, c}$ & $1.62(0.66)^{\mathrm{a}, \mathrm{b}, \mathrm{c}}$ & 0.207 & 0.310 \\
\hline \multirow{6}{*}{$\begin{array}{c}14 \\
\mathrm{~km} / \mathrm{h}\end{array}$} & $\mathrm{CT}(\mathrm{s})$ & $0.266(0.020)^{a, b, c, c, d}$ & $0.271(0.020)^{a, b, c, d}$ & $0.259(0.017)^{\mathrm{a}, \mathrm{b}, \mathrm{c}, \mathrm{c} d}$ & 0.010 & 0.650 \\
\hline & $\mathrm{FT}(\mathrm{s})$ & $0.087(0.018)^{a, b, c, c, d}$ & $0.086(0.020)^{a, b, c, d}$ & $0.088(0.017)^{\mathrm{a}, \mathrm{b}, \mathrm{c}, \mathrm{d}}$ & 0.537 & 0.108 \\
\hline & $\mathrm{SL}(\mathrm{cm})$ & $137.07\left(7.33^{a, b, c, c d}\right.$ & $138.45(7.86)^{\mathrm{a}, \mathrm{b}, \mathrm{c}, \mathrm{d}}$ & $135.17(6.15)^{\mathrm{a}, \mathrm{b}, \mathrm{c}, \mathrm{d}}$ & 0.043 & 0.471 \\
\hline & SLnormalized & $1.52(0.12)^{a, b, c, c, d}$ & $1.54(0.12)^{a, b, c, c, d}$ & $1.50(0.12)^{a, b, c, c, d}$ & 0.089 & 0.337 \\
\hline & SF (step/min) & $170.71(8.88)^{a, b, c, d}$ & $169.07(9.44)^{\mathrm{a}, \mathrm{b}, \mathrm{c}, \mathrm{d}}$ & $172.96(7.60)^{a, b, c, c, d}$ & 0.048 & 0.470 \\
\hline & $\mathrm{SA}(\stackrel{\circ}{(9)}$ & $1.62(0.66)^{\mathrm{a}, \mathrm{a}, \mathrm{c}, \mathrm{d}}$ & $1.57(0.67)^{\mathrm{a}, \mathrm{b}, \mathrm{c}, \mathrm{d}}$ & $1.68(0.64)^{\mathrm{a}, \mathrm{b}, \mathrm{c}}$ & 0.450 & 0.170 \\
\hline \multirow{6}{*}{$\begin{array}{c}15 \\
\mathrm{~km} / \mathrm{h}\end{array}$} & CT (s) & $0.254(0.019)^{\mathrm{a} a \mathrm{~b}, \mathrm{c}, \mathrm{d}, \mathrm{e}}$ & $0.258(0.019)^{\mathrm{a} a, c, d, \mathrm{~d}, \mathrm{e}}$ & $0.248(0.017)^{a, b, c, d, e}$ & 0.020 & 0.545 \\
\hline & $\mathrm{FT}(\mathrm{s})$ & $0.093(0.017)^{\mathrm{a}, \mathrm{b}, \mathrm{c}, \mathrm{d}, \mathrm{e}}$ & $0.093(0.017)^{\mathrm{a}, \mathrm{b}, \mathrm{c}, \mathrm{d}, \mathrm{e}}$ & $0.095\left(0.017^{a \mathrm{a}, \mathrm{b}, \mathrm{c}, \mathrm{d}, \mathrm{e}}\right.$ & 0.744 & 0.118 \\
\hline & $\mathrm{SL}(\mathrm{cm})$ & $144.36(7.32)^{a, b, c, d, e}$ & $146.25(7.62)^{a, b, c, d, e}$ & $141.76(6.10)^{a, b, c, d, e}$ & 0.005 & 0.580 \\
\hline & SLnormalized & $1.61(0.13)^{a, b, c, d, d, e}$ & $1.63(0.12)^{a, b, c, d, d e}$ & $1.58(0.13)^{a, b, c, d, e}$ & 0.048 & 0.401 \\
\hline & $\mathrm{SF}$ (step/min) & $173.72(8.76)^{\mathrm{a}, \mathrm{b}, \mathrm{c}, \mathrm{d}, \mathrm{e}}$ & $171.51(9.00)^{a, b, c, d, e}$ & $176.75(7.54)^{a, b, c, d, e}$ & 0.006 & 0.643 \\
\hline & SA $(\stackrel{9}{)})$ & $1.74(0.61)^{\mathrm{a}, \mathrm{b}, \mathrm{c}, \mathrm{d}, \mathrm{e}}$ & $1.74(0.62)^{a, b, c, d, e}$ & $1.74(0.60)^{a, b, c}$ & 0.980 & $<0.1$ \\
\hline \multirow{7}{*}{$\begin{array}{c}16 \\
\mathrm{~km} / \mathrm{h}\end{array}$} & CT (s) & $0.243(0.018)^{a, b, c, d, e, f}$ & $0.248(0.018)^{a, b, c, d, d, e f}$ & $0.237(0.015)^{a, b, c, d, d, f}$ & 0.006 & 0.667 \\
\hline & $\mathrm{FT}(\mathrm{s})$ & $0.097(0.017)^{\mathrm{a}, \mathrm{b}, \mathrm{b}, \mathrm{d}, \mathrm{d}, \mathrm{f}}$ & $0.098(0.017)^{a, b, c, d, e, f}$ & $0.095(0.017)^{\mathrm{a}, \mathrm{b}, \mathrm{c}, \mathrm{d}, \mathrm{e}}$ & 0.483 & 0.178 \\
\hline & $\mathrm{SL}(\mathrm{cm})$ & $150.99(8.03)^{a, b, c, d, d, f}$ & $153.45(8.27)^{a, b, c, d, d, f, f}$ & $147.64(6.37)^{a, b, c, d, d, f}$ & 0.001 & 0.794 \\
\hline & SLnormalized & $1.68(0.14)^{a, b, c, d, e, f}$ & $1.71(0.13)^{a, b, c, d, e, f}$ & $1.63(0.13)^{a, b, c, d, e, f}$ & 0.008 & 0.622 \\
\hline & SF (step/min) & $177.09(9.36)^{\mathrm{a}, \mathrm{b}, \mathrm{c}, \mathrm{d}, \mathrm{d}, \mathrm{f}}$ & $174.24(9.35)^{a, b, b, c, d, f, f}$ & $181.02(7.93)^{a, b, c, d, d, f}$ & 0.001 & 0.787 \\
\hline & $\mathrm{SA}\left({ }^{\circ}\right)$ & $1.79(0.59)^{\mathrm{a}, \mathrm{b}, \mathrm{c}, \mathrm{d}, \mathrm{e}}$ & $1.80(0.60)^{a, b, c, d, e}$ & $1.77(0.58)^{\mathrm{a}, \mathrm{b}, \mathrm{c}}$ & 0.795 & $<0.01$ \\
\hline & $\begin{array}{l}\text { CT, contact time; } F T, f \\
\text { angle; } E S: \text { Cohen's } \\
\text { indicates significant } \\
\text { differences according to } \\
(p<.05) ; \text { i indicates sig }\end{array}$ & $\begin{array}{l}\text { time; } S L, \text { step length; } S \\
\text { t size. a indicates signif } \\
n \text {-group differences ace } \\
\text { n/h values ( } p<.05 \text { ); } \\
\text { nt within-group differ } \\
\quad \text { group differences }\end{array}$ & $\begin{array}{l}\text { ralized: step length divid } \\
\text { nt within-group differe } \\
\text { ing to } 11 \mathrm{~km} / \mathrm{h} \text { values } \\
\text { cates significant within } \\
\text { es according to } 14 \mathrm{~km} / \mathrm{h} \\
\text { cording to } 15 \mathrm{~km} / \mathrm{h} \text { val }\end{array}$ & $\begin{array}{l}\text { leg length; } S F, \text { step fre } \\
\text { cording to } 10 \mathrm{~km} / \mathrm{h} \text { val } \\
\text { s); c indicates significan } \\
\text { o differences according } \\
\mathrm{s}(p<.05) ; \text { indicates si } \\
<.05)\end{array}$ & $\begin{array}{l}\text { SA: step } \\
.05) ;{ }^{b} \\
\text { l-group } \\
\text { h/h values } \\
\text { it within- }\end{array}$ & \\
\hline
\end{tabular}


Table 3

Descriptive values and association observed for the within-participant standard deviation (SD) of the spatiotemporal parameters (CT, FT, SL, SF and SA) at every running velocity tested, according to sex.

\begin{tabular}{|c|c|c|c|c|c|c|}
\hline & & Total $(\mathrm{n}=97)$ & Male $(\mathrm{n}=51)$ & Female $(n=46)$ & $p$ & ES \\
\hline \multirow{6}{*}{$\begin{array}{c}10 \\
\mathrm{~km} / \mathrm{h}\end{array}$} & CT (s) & $0.009(0.003)$ & $0.009(0.003)$ & $0.009(0.002)$ & 0.157 & $<0.1$ \\
\hline & FT (s) & $0.010(0.003)$ & $0.010(0.003)$ & $0.010(0.002)$ & 0.548 & $<0.1$ \\
\hline & $\mathrm{SL}(\mathrm{cm})$ & 3.83 (1.17) & 3.78 (1.39) & $3.91(0.78)$ & 0.632 & 0.115 \\
\hline & SLnormalized & $0.05(0.02)$ & $0.05(0.03)$ & $0.04(0.01)$ & 0.623 & 0.443 \\
\hline & SF (step/min) & $4.91(1.64)$ & $4.85(1.89)$ & $4.98(1.23)$ & 0.738 & $<0.1$ \\
\hline & $\mathrm{SA}\left(\left(^{\circ}\right)\right.$ & $0.209(0.104)$ & $0.201(0.112)$ & $0.219(0.090)$ & 0.438 & 0.178 \\
\hline \multirow{6}{*}{$\begin{array}{c}11 \\
\mathrm{~km} / \mathrm{h}\end{array}$} & CT (s) & $0.008(0.003)$ & $0.009(0.003)$ & $0.008(0.002)$ & 0.054 & 0.392 \\
\hline & FT (s) & $0.010(0.003)$ & $0.010(0.003)$ & $0.010(0.002)$ & 0.570 & $<0.1$ \\
\hline & $\mathrm{SL}(\mathrm{cm})$ & 4.39 (1.55) & $4.54(1.70)$ & 4.19 (1.32) & 0.318 & 0.230 \\
\hline & SLnormalized & $0.05(0.03)^{\mathrm{a}}$ & $0.05(0.03)^{\mathrm{a}}$ & $0.05(0.01)^{\mathrm{a}}$ & 0.156 & $<0.1$ \\
\hline & SF (step/min) & $5.24(1.90)$ & $5.37(2.10)$ & $5.06(1.59)$ & 0.457 & 0.169 \\
\hline & $\mathrm{SA}\left({ }^{\circ}\right)$ & $0.261(0.116)^{\mathrm{a}}$ & $0.259(0.135)^{\mathrm{a}}$ & $0.263(0.085)^{\mathrm{a}}$ & 0.856 & $<0.1$ \\
\hline \multirow{6}{*}{$\begin{array}{c}12 \\
\mathrm{~km} / \mathrm{h}\end{array}$} & CT (s) & $0.006(0.002)^{a, b}$ & $0.006(0.002)^{a, b}$ & $0.006(0.002)^{\mathrm{a}}$ & 0.992 & $<0.1$ \\
\hline & FT (s) & $0.011(0.003)^{\mathrm{a}}$ & $0.010(0.003)$ & $0.010(0.002)$ & 0.142 & $<0.1$ \\
\hline & $\mathrm{SL}(\mathrm{cm})$ & $4.23(1.29)$ & $4.25(1.38)$ & $4.20(1.19)$ & 0.881 & $<0.1$ \\
\hline & SLnormalized & $0.05(0.02)^{a, b}$ & $0.05(0.02)^{a, b}$ & $0.05(0.02)^{a, b}$ & 0.662 & $<0.1$ \\
\hline & SF (step/min) & 4.95 (1.49) & 4.99 (1.62) & $4.89(1.30)$ & 0.762 & $<0.1$ \\
\hline & $\mathrm{SA}\left(\left(^{\circ}\right)\right.$ & $0.321(0.107)^{\mathrm{a}, \mathrm{b}}$ & $0.327(0.125)^{a, b}$ & $0.313(0.075)^{\mathrm{a}}$ & 0.556 & 0.135 \\
\hline \multirow{6}{*}{$\begin{array}{c}13 \\
\mathrm{~km} / \mathrm{h}\end{array}$} & CT (s) & $0.006(0.002)^{a, b}$ & $0.006(0.002)^{a, b}$ & $0.006(0.002)^{a, b}$ & 0.257 & $<0.1$ \\
\hline & FT (s) & $0.010(0.003)$ & $0.010(0.003)$ & $0.010(0.002)$ & 0.095 & $<0.1$ \\
\hline & $\mathrm{SL}(\mathrm{cm})$ & 4.35 (1.49) & $4.58(1.63)^{\mathrm{a}}$ & $4.02(1.23)$ & 0.094 & 0.389 \\
\hline & SLnormalized & $0.05(0.02)^{a, b, c}$ & $0.05(0.02)^{a, b, c}$ & $0.05(0.02)^{a, b, c}$ & 0.110 & $<0.1$ \\
\hline & $\mathrm{SF}$ (step/min) & $4.80(1.60)$ & $5.18(1.79)$ & 4.69 (1.27) & 0.967 & 0.316 \\
\hline & $\mathrm{SA}\left({ }^{\circ}\right)$ & $0.334(0.110)^{a, b}$ & $0.336(0.116)^{a, b}$ & $0.332(0.103) a$ & 0.869 & $<0.1$ \\
\hline \multirow{6}{*}{$\begin{array}{c}14 \\
\mathrm{~km} / \mathrm{h}\end{array}$} & $\mathrm{CT}(\mathrm{s})$ & $0.006(0.002)^{a, b}$ & $0.007(0.002)^{a, b}$ & $0.006(0.002)^{a, b}$ & 0.099 & 0.505 \\
\hline & FT (s) & $0.092(0.003)^{c}$ & $0.010(0.003)$ & $0.009(0.002)^{a, b, c}$ & 0.068 & 0.392 \\
\hline & $\mathrm{SL}(\mathrm{cm})$ & 4.49 (1.61) & $4.70(1.81)^{\mathrm{a}}$ & $4.20(1.25)$ & 0.163 & 0.322 \\
\hline & SLnormalized & $0.05(0.02)^{a, b, c, d}$ & $0.05(0.02)^{a, b, c, d}$ & $0.05(0.02)^{a, b, c, d}$ & 0.260 & $<0.1$ \\
\hline & SF (step/min) & $4.98(1.73)$ & $5.25(2.01)$ & $4.61(1.23)$ & 0.165 & 0.383 \\
\hline & $\mathrm{SA}\left(\left(^{\circ}\right)\right.$ & $0.295(0.089)^{\mathrm{a}, \mathrm{d}}$ & $0.301(0.089)^{\mathrm{a}}$ & $0.285(0.089)$ & 0.421 & 0.182 \\
\hline \multirow{6}{*}{$\begin{array}{c}15 \\
\mathrm{~km} / \mathrm{h}\end{array}$} & $\mathrm{CT}(\mathrm{s})$ & $0.006(0.002)^{a, b}$ & $0.006(0.002)^{a, b}$ & $0.005(0.002)^{a, b}$ & 0.017 & 0.505 \\
\hline & FT (s) & $0.009(0.003)^{c}$ & $0.010(0.003)$ & $0.008(0.002)^{a, b, c}$ & 0.007 & 0.784 \\
\hline & $\mathrm{SL}(\mathrm{cm})$ & $4.41(1.68)$ & $4.78(1.90)^{\mathrm{a}}$ & 3.91 (1.17) & 0.019 & 0.550 \\
\hline & SLnormalized & $0.05(0.02)^{a, b, b, c, d, e}$ & $0.05(0.02)^{a, b, b, c, d, e}$ & $0.05(0.01)^{a, b, c, c, d, e}$ & 0.031 & $<0.1$ \\
\hline & SF (step/min) & $5.14(1.54)$ & $5.45(1.55)$ & $4.62(1.23)$ & 0.102 & 0.595 \\
\hline & $\mathrm{SA}\left(\left(^{\circ}\right)\right.$ & $0.296(0.099)^{\mathrm{a}, \mathrm{d}}$ & $0.315(0.100)^{\mathrm{a}, \mathrm{b}}$ & $0.269(0.093)^{\mathrm{d}}$ & 0.039 & 0.476 \\
\hline \multirow{6}{*}{$\begin{array}{c}16 \\
\mathrm{~km} / \mathrm{h}\end{array}$} & $\mathrm{CT}(\mathrm{s})$ & $0.006(0.002)^{a, b}$ & $0.006(0.002)^{a, b}$ & $0.005(0.002)^{a, b}$ & 0.150 & 0.505 \\
\hline & FT (s) & $0.009(0.003)^{c}$ & $0.010(0.003)$ & $0.008(0.002)^{a, b, c}$ & 0.002 & 0.784 \\
\hline & $\mathrm{SL}(\mathrm{cm})$ & $4.72(1.87)^{\mathrm{a}}$ & $5.14(2.16)^{\mathrm{a}}$ & 4.15 (1.18) & 0.015 & 0.567 \\
\hline & SLnormalized & $0.05(0.02)^{a, b, c, c, e, e, f}$ & $0.06(0.02)^{a, b, c, d, d, e, f}$ & $0.05(0.01)^{a, b, c, d, d, e, f}$ & 0.014 & 0.629 \\
\hline & SF (step/min) & $5.15(1.55)$ & $5.45(1.55)$ & $4.73(1.46)$ & 0.036 & 0.482 \\
\hline & $S A(\stackrel{o}{)})$ & $0.285(0.077)^{a, c, d}$ & $0.302(0.071)^{a}$ & $0.261(0.078)^{d}$ & 0.016 & 0.560 \\
\hline
\end{tabular}

CT, contact time; FT, flight time; SL, step length; SLnormalized: step length divided into leg length; SF, step frequency; SA: step angle; ES: Cohen' $d$ effect size. ${ }^{a}$ indicates significant within-group differences according to $10 \mathrm{~km} / \mathrm{h}$ values $(p<.05) ;{ }^{b}$ indicates significant within-group differences according to $11 \mathrm{~km} / \mathrm{h}$ values $(p<.05)$; c indicates significant within-group differences according to $12 \mathrm{~km} / \mathrm{h}$ values $(p<.05)$; d

indicates significant within-group differences according to $13 \mathrm{~km} / \mathrm{h}$ values $(p<.05)$; e indicates significant within-group differences according to $14 \mathrm{~km} / \mathrm{h}$ values $(p<.05)$; $f$ indicates significant within-group differences according to $15 \mathrm{~km} / \mathrm{h}$ values $(p<.05)$ 
The results obtained in the current study reinforce previous studies' findings regarding spatiotemporal adaptations with increased running velocity. A previous study showed that an increase of $2 \mathrm{~km} / \mathrm{h}$ in running speed increased SF ( $\sim 7 \mathrm{step} / \mathrm{min})$, SL $(\sim 0.3 \mathrm{~m})$, and decreased CT ( $\sim 0.02 \mathrm{~ms})$, independently of the type of the foot strike pattern (Ogueta-Alday et al., 2014). It seems clear that to run faster, FT needs to be increased and CT needs to be decreased to aid repositioning the legs during running (Brughelli et al., 2010). Based on that relationship, SF also needs to be increased to run faster (Morin et al., 2007). It has also been suggested that SL increases linearly with running velocity up to $25 \mathrm{~km} / \mathrm{h}$ (Brughelli et al., 2010), which is in consonance with our findings (SL increased over the protocol up to 16 $\mathrm{km} / \mathrm{h}$ ). Of note, these changes are not important only in terms of athletic performance, but also from an injury point of view. Changes in spatiotemporal parameters during running may affect impact shock (Heiderscheit et al., 2011) which might be associated to risk of injury (Lenhart et al., 2014), even though more research is clearly needed to highlight the relationship between running-related injuries and biomechanical risk factors (Ceyssens et al., 2019).

As the information available on SA during running is limited, comparisons are difficult. However, Santos-Concejero et al. (2014) point to SA as an easily obtainable measure that reveals greater potential for running performance and economy than other biomechanical variables. The current study shows that the SA increases with an increased running velocity and this finding is in accordance with the results reported in previous studies (Roche-Seruendo et al., 2018; Santos-Concejero et al., 2014). This adaptation may be a marker of the athlete's ability to efficiently maximize FT and minimize CT with effective energy transfer during ground contact. Greater SA would lead athletes to experience shorter $\mathrm{CT}$, allowing better running economy (Santos-Concejero et al., 2014). As indicated by a previous study (Santos-Concejero et al., 2014), this phenomenon could be due to an early contraction of the muscles involved in the stride during the stance phase, leading the centre of mass to be projected forward with greater efficiency.

Whereas the influence of running velocity on spatiotemporal gait characteristics has been previously examined in the literature (Brughelli et al., 2010; Ogueta-Alday et al., 2014; Padulo et al., 2012; Roche-Seruendo et al., 2018), the evidence about the influence of running velocity on step variability is limited. In this regard, an important consideration is the measure selected to examine step variability (i.e., within-participant $\mathrm{SD}$ ). Since previous studies have used indistinctly the SD (Paquette et al., 2017) or coefficient of variation (Nakayama et al., 2010), caution must be taken when comparing data between studies. According to a previous study (Jordan and Newell, 2008), step variability seems to be speed-dependent, and current results provide support to that statement. In this context, and considering that step variability seems to be related to both injuries (Hamill et al., 2012; Meardon et al., 2011) and endurance performance (Nakayama et al., 2010), further research is clearly needed to highlight the role of step variability in endurance running. The current work provides some insights into the dynamics of step variability with increasing running velocity, and it warns about the influence of running velocity on it.

Regarding sex comparisons, differences were reported in some spatiotemporal parameters and step variability. Females showed shorter CT than males at every velocity tested, shorter SL and SLnormalized at velocities $\geq 14 \mathrm{~km} / \mathrm{h}$, and higher SF, with no differences in the SA. Likewise, betweensex differences were found in step variability at $15-16 \mathrm{~km} / \mathrm{h}$, with males showing greater SD than females in each parameter. Only one previous study has directly examined sex-differences in spatiotemporal parameters during running (Roche-Seruendo et al., 2019), although without a step variability comparison. The authors (RocheSeruendo et al., 2019) examined the influence of sex and anthropometric characteristics of amateur endurance runners during running at a constant velocity (i.e., $12 \mathrm{~km} / \mathrm{h}$ ), with no between-sex differences found in spatiotemporal parameters. Even though the current finding seems to contrast with those of the aforementioned study, the results are actually consistent. Both works reported longer CT in males than in females $(+0.11$ $\mathrm{s}$ in the current study and $+0.012 \mathrm{~s}$ in the aforementioned study, Roche-Seruendo et al., 2019) during running on a treadmill at $12 \mathrm{~km} / \mathrm{h}$.

The lack of research covering this topic makes comparison difficult. Previous studies 
found no significant differences in the foot strike pattern in adult male and female endurance runners (Latorre-Román et al., 2015), in 6-16 years old (Latorre-Román et al., 2017) and 3-6 years old participants (Latorre-Román et al., 2018). However, the current study is the first one to address spatiotemporal differences between sexes. Moreover, while some studies did not normalize the spatiotemporal parameters according to the body size of runners (Sakaguchi et al., 2014), others did (Roche-Seruendo et al., 2019; Takabayashi et al., 2017). It is well-known that (and our data support it) female runners are generally smaller in body size than males (RocheSeruendo et al., 2019), which may affect the preferred movement patterns and spatiotemporal adaptations at increased running speeds. Considering the lack of evidence regarding the sex effect on spatiotemporal gait characteristics during running, the current study highlights the dynamics of traditional (i.e., CT, FT, SL and SF) and recently-incorporated parameters (SA and step variability) in both male and female endurance athletes by analyzing the differences.

Some limitations need to be addressed. First, the incremental running protocol considered running velocities up to $16 \mathrm{~km} / \mathrm{h}$, without information about faster running velocities. Nevertheless, the performance level of participants (amateur runners) did not allow to progress into higher running speeds, not without the risk of fatigue. Second, a biomechanical analysis including joint angles and kinetic variables was not possible. Third, the foot strike pattern was not controlled in this study. Rearfoot-, midfoot- and forefoot-strike runners may have different running biomechanics, thus potentially different response to increased running velocities. Notwithstanding these limitations, the current work provides a detailed description of spatiotemporal parameters response over a range of common velocities for training in a sample of 97 amateur endurance runners, considering the role of sex on these spatiotemporal adaptations. Today, many devices provide information, even real-time feedback, about spatiotemporal parameters during running. Having some values to compare with at common running velocities, and for both sexes, may be of interest for coaches and athletes.

In conclusion, increasing running velocity $(10-16 \mathrm{~km} / \mathrm{h})$ makes CT shorter, FT and SL longer, and SF and SA greater in amateur endurance runners, inducing changes in step variability. Additionally, CT and SL were shorter, while FT and SF were greater in females, whereas step variability (SD in each spatiotemporal parameter at $15-16 \mathrm{~km} / \mathrm{h}$ ) was greater in males.

\section{Acknowledgements}

The authors would like to thank to all the participants.

\section{References}

Alexander RM. Stride length and speed for adults, children, and fossil hominids. Am J Phys Anthrop, 1984 https://doi.org/10.1002/ajpa.1330630105

Belli A, Lacour JR, Komi PV, Candau R, Denis C. Mechanical step variability during treadmill running. Eur J Appl Physiol Occup Physiol, 1995; 70(6): 510-517

Brown AM, Zifchock RA, Hillstrom HJ. The effects of limb dominance and fatigue on running biomechanics. Gait Post, 2014; 39(3): 915-919. https://doi.org/10.1016/j.gaitpost.2013.12.007

Brughelli M, Cronin J, Chaouachi A. Effects of running velocity on running kinetics and kinematics. J Strength Cond Res, 2010; 24(0): 1-7

Ceyssens L, Vanelderen R, Barton C, Malliaras P, Dingenen B. Biomechanical Risk Factors Associated with Running-Related Injuries: A Systematic Review. Sports Medic, 2019; 1-21. https://doi.org/10.1007/s40279-019-01110-z

Cohen J. Statistical power analysis for the behavioral sciences. Statistical Power Analysis for the Behavioral Sciences (Vol. 2nd), 1988 
García-Pinillos F, Latorre-Román PA, Ramírez-Campillo R, Párraga-Montilla JA, Roche-Seruendo LE. Minimum time required for assessing step variability during running at submaximal velocities. $J$ Biomech, 2018a; in press https://doi.org/10.1016/J.JBIOMECH.2018.09.005

García-Pinillos F, Latorre-Román PÁ, Ramírez-Campillo R, Párraga-Montilla JA, Roche-Seruendo LE. How does the slope gradient affect spatiotemporal parameters during running? Influence of athletic level and vertical and leg stiffness. Gait Post, 2018b; 68: 72-77. https://doi.org/10.1016/J.GAITPOST.2018.11.008

Hamill J, Palmer C, Van Emmerik RE. Coordinative variability and overuse injury. Sports Med, Arthr, Rehab, Ther Techn, 2012; 4(1), 45

Heiderscheit BC, Chumanov ES, Michalski MP, Wille CM, Ryan MB. Effects of step rate manipulation on joint mechanics during running. Med Sci Sports Exerc, 2011; 43(2): 296-302

Heise GD, Martin PE. Are variations in running economy in humans associated with ground reaction force characteristics? Eur J Appl Physiol, 2001; 84(5): 438-442

Jordan K, Challis JH, Cusumano JP, Newell KM. Stability and the time-dependent structure of gait variability in walking and running. Hum Mov Sci, 2009; 28(1): 113-128. https://doi.org/10.1016/j.humov.2008.09.001

Jordan K, Newell KM. The Structure of Variability in Human Walking and Running is Speed-Dependent. Exerc Sport Sci Rev, 2008; 36(4), 200-204

Kong PW, de Heer H. Anthropometric, gait and strength characteristics of kenyan distance runners. J Sports Sci Med, 2008; 7(4): 499-504

Latorre-Román PÁ, Muñoz-Jiménez M, Soto-Hermoso VM, Sánchez JS, Molina AM, Robles-Fuentes A, García-Pinillos F. Acute effect of a long-distance road competition on foot strike patterns, inversion and kinematics parameters in endurance runners. Int J Perf Anal in Sport, 2015; 15: 588-597

Latorre-Román PÁ, Párraga-Montilla JA, Guardia-Monteagudo I, García-Pinillos F. Foot strike pattern in preschool children during running: sex and shod-unshod differences. European J Sport Sci, 2018; 17(3): 407-414. https://doi.org/10.1080/17461391.2017.1422545

Latorre-Román PÁ, Balboa FR, García-Pinillos F. Foot strike pattern in children during shod-unshod running. Gait Post, 2017; 58: 220-222. https://doi.org/10.1016/j.gaitpost.2017.07.121

Lavcanska V, Taylor NF, Schache AG. Familiarization to treadmill running in young unimpaired adults. Hum Mov Sci, 2005; 24(4): 544-557. https://doi.org/10.1016/j.humov.2005.08.001

Lee MM, Song CH, Lee KJ, Jung SW, Shin DC, Shin SH. Concurrent Validity and Test-retest Reliability of the OPTOGait Photoelectric Cell System for the Assessment of Spatio-temporal Parameters of the Gait of Young Adults. J Phys Ther Sci, 2014; 26(1): 81-85. https://doi.org/10.1589/jpts.26.81

Lenhart RL, Thelen D, Heiderscheit B. Hip muscle loads during running at various step rates. J Orthop Sports Phys Ther, 2014; 44(10): 766-774

Meardon SA, Hamill J, Derrick TR. Running injury and stride time variability over a prolonged run. Gait Post, 2011; 33(1): 36-40. https://doi.org/10.1016/j.gaitpost.2010.09.020

Morin JB, Samozino P, Zameziati K, Belli A. Effects of altered stride frequency and contact time on leg-spring behavior in human running. J Biomech, 2007; 40(15): 3341-3348

Nakayama Y, Kudo K, Ohtsuki T. Variability and fluctuation in running gait cycle of trained runners and non-runners. Gait Post, 2010; 31(3): 331-335. https://doi.org/10.1016/j.gaitpost.2009.12.003

Ogueta-Alday A, Rodríguez-Marroyo JA, García-López J. Rearfoot striking runners are more economical than midfoot strikers. Med Sci Sports Exerc, 2014; 46(3): 580-585. https://doi.org/10.1249/MSS.0000000000000139

Padulo J, Annino G, Migliaccio GM, D’Ottavio S, Tihanyi J. Kinematics of Running at Different Slopes and Speeds. J Strength Cond Res, 2012. https://doi.org/10.1519/JSC.0b013e318231aafa

Paquette MR, Milner CE, Melcher DA. Foot contact angle variability during a prolonged run with relation to injury history and habitual foot strike pattern. Scand J Med Sci Sports, 2017; 27(2): 217-222. https://doi.org/10.1111/sms.12647

Roche-Seruendo LE, García-Pinillos F, Haicaguerre J, Bataller-Cervero AV, Soto-Hermoso VM, LatorreRomán PÁ. Lack of influence of muscular performance parameters on spatio-temporal adaptations with increased running velocity. J Strength Cond Res, 2018; 32(2): 409-415. 
https://doi.org/10.1519/JSC.0000000000001845

Roche-Seruendo LE, García-Pinillos F, Auria-Martin I, Bataller-Cervero AV, Latorre Román PA, SotoHermoso VM. Effects of different percentages of body weight support on spatiotemporal step characteristics during running. J Sports Sci, 2017; 1-6. https://doi.org/10.1080/02640414.2017.1394584

Roche-Seruendo LE, Latorre-Román PÁ, Soto-Hermoso VM, García-Pinillos F. Do sex and body structure influence spatiotemporal step characteristics in endurance runners? Sci Sports, 2019; 1: 1-8.

Sakaguchi M, Ogawa H, Shimizu N, Kanehisa H, Yanai T, Kawakami Y. Gender differences in hip and ankle joint kinematics on knee abduction during running. Eur J Sport Sci, 2014; 14 Suppl 1(sup1): 302-309. https://doi.org/10.1080/17461391.2012.693953

Santos-Concejero J, Tam N, Granados C, Irazusta J, Bidaurrazaga-Letona I, Zabala-Lili J, Gil SM. Stride angle as a novel indicator of running economy in well-trained runners. J Strength Cond Res, 2014; 28(7): 18891895

Takabayashi T, Edama M, Nakamura M, Nakamura E, Inai T, Kubo M. Gender differences associated with rearfoot, midfoot, and forefoot kinematics during running. Eur J Sport Sci, 2017; 17(10): 1289-1296. https://doi.org/10.1080/17461391.2017.1382578

\section{Corresponding author:}

\section{Felipe García-Pinillos, PhD}

Department of Physical Education, Sports and Recreation.

Universidad de La Frontera (Temuco, Chile). Calle Uruguay, 1980 (Temuco, Chile),

E-mail: fegarpi@gmail.com 